\title{
Non-transmural myocardial infarction associated with regional contractile function is an independent predictor of positive
} outcome: an integrated approach to myocardial
viability

Gianluca Di Bella ${ }^{1}$, Giovanni Donato Aquaro ${ }^{2}$, Jan Bogaert ${ }^{3}$, Paolo Piaggi ${ }^{4}$, Antonio Micari ${ }^{1}$, Fausto Pizzino ${ }^{5}$, Giovanni Camastra ${ }^{6}$, Scipione Carerj ${ }^{1}$, Mariapaola Campisi ${ }^{1}$, Antonio Bracco ${ }^{1,9}$, Maria Ludovica Carerj ${ }^{1}$, Michele Emdin², Bijoy K. Khandheria ${ }^{7^{*}}(1)$ and Alessandro Pingitore ${ }^{8}$

\begin{abstract}
Background: Cardiovascular magnetic resonance permits assessment of irreversible myocardial fibrosis and contractile function in patients with previous myocardial infarction. We aimed to assess the prognostic value of myocardial fibrotic tissue with preserved/restored contractile activity.

Methods: In 730 consecutive myocardial infarction patients (64 \pm 11 years), we quantified left ventricular (LV) end-diastolic (EDV) and end-systolic (ESV) volumes, ejection fraction (EF), regional wall motion (WM) (1 normal, 2 hypokinetic, 3 akinetic, 4 dyskinetic), and WM score index (WMSI), and measured the transmural (1-50 and 51-100) and global extent of the infarct scar by late gadolinium enhancement (LGE). Contractile fibrotic (CT-F) segments were identified as those showing WM- 1 and WM-2 with LGE $\leq$ or $\geq 50 \%$.

Results: During follow-up (median 2.5, range 1-4.7 years), cardiac events (cardiac death or appropriate implantable defibrillator shocks) occurred in 123 patients (17\%). At univariate analysis, age, LVEDV, LVESV, LVEF, WMSI, extent of LGE, segments with transmural extent $>50 \%$, and CT-F segments were associated with cardiac events. At multivariate analysis, age $>65$ years, LVEF $<30 \%, \mathrm{WMSI}>1.7$, and dilated LVEDV independently predicted cardiac events, while CT-F tissue was the only independent predictor of better outcome. After adjustment for LVEF $<30 \%$ and LVEDV dilatation, the presence of CT-F tissue was associated with good prognosis.
\end{abstract}

Conclusions: In addition to CMR imaging parameters associated with adverse outcome (severe LV dysfunction, poor WM, and dilated EDV), the presence of fibrotic myocardium showing contractile activity in patients with previous myocardial infarction yields a beneficial effect on patient survival.

Keywords: Contractile segmental function, Myocardial infarction, Myocardial viability

*Correspondence: publishing22@aurora.org

${ }^{7}$ Aurora Cardiovascular and Thoracic Services, Advocate Aurora Health,

Aurora Sinai/Aurora St. Luke's Medical Centers, 2801 W. Kinnickinnic River Parkway, Ste. 880, Milwaukee, WI 53215, USA

Full list of author information is available at the end of the article

\section{Background}

Left ventricular (LV) dysfunction is a common finding after myocardial infarction (MI) that is associated with high morbidity and mortality in patients with heart failure $[1,2]$. It is not only the extent of myocardial necrosis original author(s) and the source, provide a link to the Creative Commons licence, and indicate if changes were made. The images or other third party material in this article are included in the article's Creative Commons licence, unless indicated otherwise in a credit line to the material. If material is not included in the article's Creative Commons licence and your intended use is not permitted by statutory regulation or exceeds the permitted use, you will need to obtain permission directly from the copyright holder. To view a copy of this licence, visit http://creativecommons.org/licenses/by/4.0/. The Creative Commons Public Domain Dedication waiver (http://creativeco mmons.org/publicdomain/zero/1.0/) applies to the data made available in this article, unless otherwise stated in a credit line to the data. 
(subendocardial and/or transmural) and the severity of myocardial dysfunction in the jeopardized myocardium that contribute to post-MI ventricular remodeling, but other pathophysiological pathways (e.g., particularly neuroendocrine systems) involving the remote zones [3]. In this clinical context, contractile function represents a key element in counteracting post-ischemic remodeling and avoiding progression of LV dysfunction and heart failure [4].

Contractile function is synonymous with myocardial viability, although several definitions exist based on the markers used by different cardiac imaging techniques: wall thickness (echocardiography and cine cardiovascular magnetic resonance [CMR]), myocardial contractile reserve (dobutamine stress echocardiography/CMR), myocyte cellular integrity (single-photon emission computed tomography (SPECT)), myocardial metabolism (positron emission tomography (PET)), and myocardial scar/fibrosis (late gadolinium enhancement [LGE]) [4-9]. It is notable that CMR provides the opportunity to assess both the transmural extent of irreversible myocardial damage and contractile function. These two variables often are intermingled as they can coexist within the same myocardial segment, and, importantly, the likelihood of improvement in contractility after revascularization is predicted by the transmural extent of the scar, i.e., the lower the transmural extent of myocardial scar tissue, the higher the probability of functional recovery [10].

Therefore, although the clinical and prognostic impact of the different markers of myocardial viability have been extensively investigated, it is still unknown whether partially scarred segments showing contractile activity yield a prognostic value. The aim of this observational, multicenter study was to analyze the prognostic value of partially scarred myocardial segments yielding contractile activity and the potential additive impact in respect to the other predictors of adverse outcome in patients with previous MI.

\section{Methods \\ Patients}

We studied 862 consecutive patients with clinical evidence of a prior MI ( $>90$ days) and a clinical indication for CMR, i.e., quantification of LV function and volumes and identification of LV thrombi, extent of scar tissue, and myocardial viability. Previous MI was documented by clinical record, Q-waves at electrocardiography (ECG), and angiographic evidence of coronary artery stenosis (luminal diameter reduced by $\geq 50 \%$ in the left main stem and $>70 \%$ stenosis in a major coronary vessel). All patients were in stable clinical condition at enrollment. Patients were excluded if they had: (1) unstable angina or recent evidence of myocardial ischemia (absence of elevated troponin) $(\mathrm{n}=57,7 \%)$; (2) prosthetic annuloplasty and/or prosthetic heart valve $(\mathrm{n}=24,3 \%)$; (3) hypertrophic cardiomyopathy $(\mathrm{n}=14,2 \%)$; (4) history of malignancy and/or prior chemotherapy treatment $(\mathrm{n}=16,2 \%)$; (5) low-quality (non-diagnostic images) or incomplete CMR scan $(\mathrm{n}=21,3 \%)$. We also excluded patients with contraindications for CMR, irregular heart rhythm, and New York Heart Association class IV because of the difficulty of performing a diagnostic CMR scan. Eventually, 730 patients $(85 \%)$ were included in the final stage of the study. Patients were studied either as outpatients $(n=160)$ or during hospitalization $(n=570)$. Clinical variables were collected before the CMR scan.

Institutional internal review boards of the involved hospitals approved our observational and retrospective study; the investigation complies with the principles outlined in the Declaration of Helsinki. All patients gave informed consent before being enrolled.

\section{CMR data acquisition}

CMR was performed using a $1.5 \mathrm{~T}$ whole-body CMR scanner (CVi, HD release, GE Medical Systems, Milwaukee, Wisconsin, USA; Gyroscan NT, Philips Healthcare, Amsterdam, the Netherlands).

According to the protocols recommended by the Society for Cardiovascular Magnetic Resonance, a breath-hold balanced steady-state free-precession ECG triggered sequence was used to evaluate global LV function. LGE images were obtained 10-20 min after a bolus injection of gadolinium-based contrast medium $(0.1 \mathrm{mmol} / \mathrm{kg}$ of gadolinium-BOPTA, Multihance, Bracco, Milan, Italy; $0.2 \mathrm{mmol} / \mathrm{kg}$ of gadoliniumDOTA, Dotarem, Guerbet, Roissy, France; or $0.2 \mathrm{mmol} /$ $\mathrm{kg}$ of gadobutrol, Gadovist, Bayer AG, Basel, Switzerland) using fast gradient-echocardiography inversion recovery sequences. Inversion time was individually adapted to null the signal of remote myocardium (usual range 220-300 ms). Cine and LGE images were acquired in at least one set of matched views including a contiguous LV short-axis and one vertical and one horizontal long-axis view.

\section{CMR data analysis}

All CMR data were analyzed off-line using commercial software (Mass, Medis, Leiden, The Netherlands). CMR studies were centrally analyzed in a core-lab with consensus among 3 experienced observers ( $>5$ years' experience) who were blinded to the clinical results and follow-up data.

Endocardial and epicardial LV contours were drawn on short-axis cine images at end-diastole and end-systole. Papillary muscle and trabeculations were considered part of the cavity. From these contours, LV end-diastolic 
volume (LVEDV), LV end-systolic volume (LVESV), LV ejection fraction (LVEF), and LV mass were calculated. Volumetric measures were normalized to body surface area [11]. A 17-segment model was used to describe LV segmental wall motion (WM). The WM of each segment was graded semiquantitatively ( 1 normal, 2 hypokinetic, 3 akinetic, 4 dyskinetic), and WM score index (WMSI) was then derived (sum of semiquantitative WM divided by 17 ).

The total extent of myocardial LGE was determined semi-automatically, using a cut-off of 5 standard deviations (SD) above the average of remote myocardium. If needed, contours were manually corrected $[12,13]$. The transmural extent of LGE was semiquantitatively measured in each segment and expressed as a percentage of the total thickness of the wall segment; it was subsequently clustered as segments with subendocardial (partially scarred) LGE (involving $1-50 \%$ of the segment wall thickness) and transmural (scarred) LGE (>50\% of the segment wall thickness) [10]. Next, the cine and LGE findings per segment were compared. Contractile fibrotic (CT-F) segments were defined as myocardial segments showing WM-1 and evidence of scarring (i.e., LGE $\leq 50 \%$ or LGE $>50 \%$ ) and/or segments having WM-2 and LGE $>50 \%$ (Fig. 1). To assess intraobserver reproducibility, the same reader repeated this assessment in 100 cases with one week between readings; interobserver reproducibility was determined after a second experienced observer, blinded to the readings by the first observer, made an assessment.

\section{Follow-up}

Follow-up was performed using a questionnaire administered to the physician during periodic ambulatory workup at our institutions (613 patients, $84 \%$ ) or by telephone call (117 patients, 16\%). This questionnaire was administered every year. Cardiac events (CEs) were defined as cardiac death and appropriate implantable cardioverter-defibrillator (ICD) shock. Appropriate ICD shock was considered equivalent to cardiac death. The cause of death was documented from medical records or death certificates. The definition of cardiac death required the documentation of life-threatening arrhythmia or cardiac arrest, or death attributable to heart failure or MI in the absence of any other precipitating factor. In the case of out-of-hospital death not followed

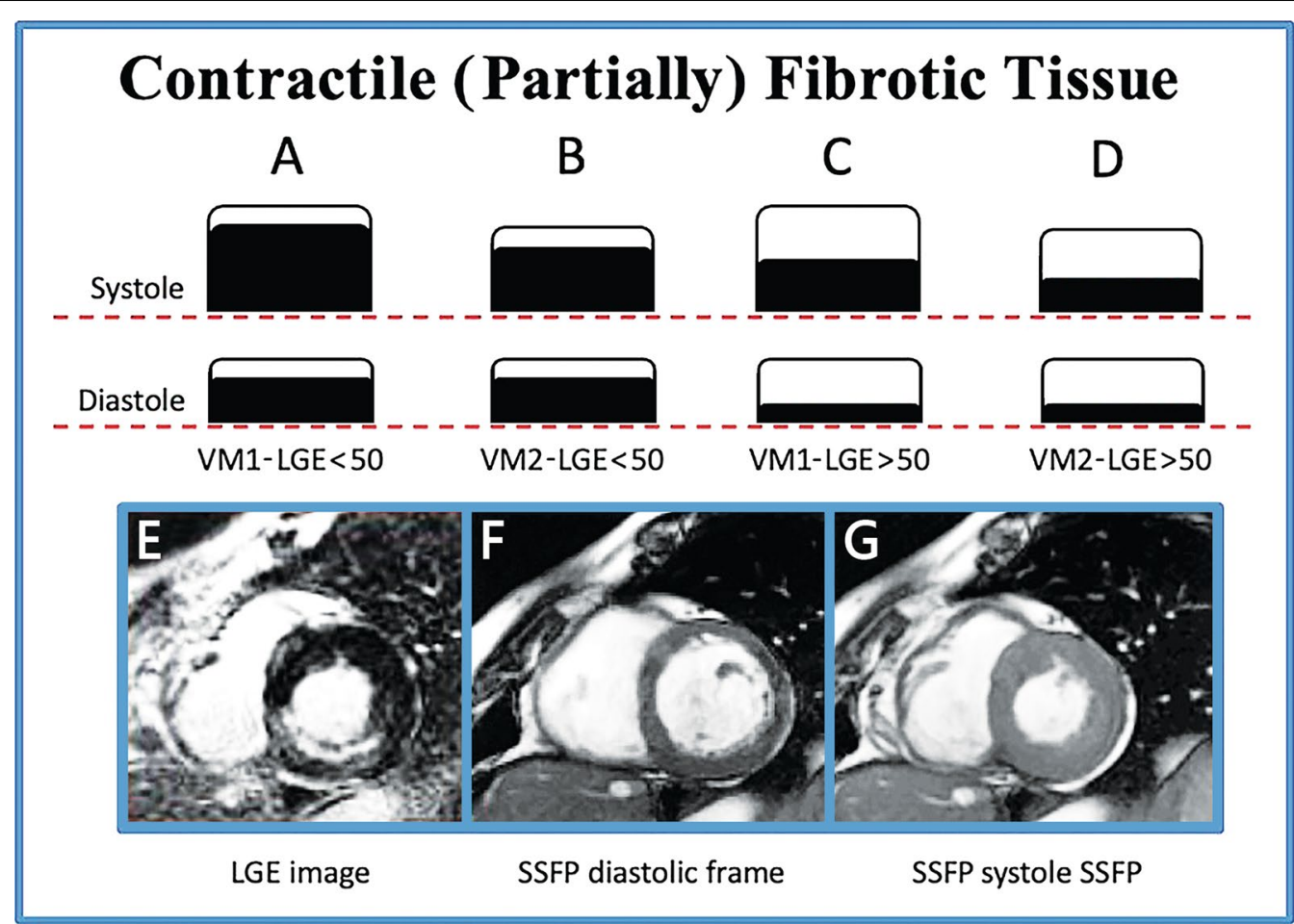

Fig. 1 Example of illustration of contractile (partially) fibrotic (CT-F) segments. CT-F segments were identified as those showing wall motion (WM-1) and late gadolinium enhancement $(\mathrm{LGE})<50 \%(\mathbf{A}), \mathrm{WM}-2$ and $\mathrm{LGE}<50 \%(\mathbf{B}), \mathrm{WM}-1$ and $\mathrm{LGE}>50 \%(\mathbf{C})$, or WM-2 and LGE $>50 \%$ (D). Panels E-G show an example CT-F segment. Hyper-enhancement area (E, LGE <50\%) in basal inferior segment associated with a good contractility (WM1) comparing diastolic frame $\mathbf{( F )}$ and systolic frame $(\mathbf{G})$ on cine cardiovascular magnetic resonance images. CT-F, contractile fibrotic; LGE, late gadolinium enhancement; SSFP, steady-state free precession; WM, wall motion 
by an autopsy, a sudden, unexpected death was considered cardiac death. ICD shocks were appropriate if triggered by malignant arrhythmia (ventricular tachycardia above the programmed cut-off of the ICD [12 intervals at 180 beats $/ \mathrm{min}$ ] or ventricular fibrillation). A complete interrogation of the ICD was performed by the referring physician in order to confirm the appropriateness of the shock [14].

Post-CMR coronary revascularization (either percutaneous or surgical) also was recorded. Coronary artery disease events included MI and post-CMR coronary revascularization.

\section{Statistical analysis}

Data analyses were performed using SPSS (version 21, Statistical Package for the Social Sciences, International Business Machines, Inc., Armonk, New York, USA). Statistical significance was considered $P<0.05$; all statistical tests were two-sided. Continuous variables were expressed as mean \pm SD or median (25th; 75th percentiles) as appropriate; categorical variables were expressed as number and percentage. The comparison between continuous variables in patients with and without CEs was performed by Student's independent samples t-test (Gaussian variables) or Wilcoxon test (skewed variables). The comparison between categorical variables was performed either by the chi-squared test or by the Fisher's exact test if an expected cell count was $<5$. The correlation between continuous variables was quantified by Pearson's correlation coefficient.

The inter- and intraobserver analysis of CT-F segments was performed by calculating the intraclass correlation coefficient (ICC).

LVEDV and LVEF were dichotomized according to validated cut-offs. In post-hoc analysis, median value was used to discriminate large extent/involvement of WMSI (>1.7), LGE extent (>13\%), and number of segments with LGE $>50 \%$ ( $\geq 3$ segments).

Univariate Cox regression analysis was used to determine which variables were associated with CEs. In multivariate stepwise analysis using the forward selection technique based on the Wald statistic, all significant continuous or dichotomous variables at univariate analysis $(P<0.05)$ were included as potential predictors. Given that LVEDV and LVEF were strongly interrelated $(\mathrm{r}=-0.70)$, two multivariate models including either LVEF (model 1) or LVEDV (model 2) were computed separately; conversely, both WMSI and LGE were included in the same multivariate model as there was no substantial collinearity (tolerance $>0.6$ ) that might have affected estimation.

On the basis of the global chi-squared statistic of each multivariate model, a risk score was computed based on the additive value of each significant dichotomous predictor in the final model. Specifically, each predictor contributed to the risk score with a weight proportional to the additive chi-squared statistic calculated in the stepwise analysis.

We used absence of CT-F (CT-F tissue not observed) instead of presence of CT-F to parallel the prognostic meaning of the other variables included in the risk score calculation.

The Kaplan-Meier survival function was used to represent the free-event survival in the population.

\section{Results}

The median interval between $\mathrm{MI}$ and CMR was 7.5 months (IQR 3-80). Patients with CEs were older $(P=0.002)$, more likely to use diuretic and aldosterone receptor antagonist therapies $(P<0.001)$, and more likely to have $>1 \mathrm{MI}(P=0.005)$ (Table 1$)$. The CE group had higher LV volumes and WMSI and lower LVEF (all $P<0.001)$. The amount of scarring was greater in the CE group $(P=0.003)$, and these patients had significantly more LGE segments, in particular, segments with LGE $>50 \%(P=0.002)$.

During follow-up, a new coronary event was observed in $212(29 \%)$ of 730 patients. Of these, 62/197 coronary events (31\%) were in patients with absence of CT-F and $150 / 533(28 \%)$ in patients with presence of CT-F $(P=0.33)$.

A total of 1429 of 12,410 segments (11\%) were defined as CT-F. Of these segments, most were WM-1 and LGE $<50 \%$ (207 segments, 14\%) or WM-2 and LGE $<50 \%$ (337 segments, 24\%), 500 (35\%) were WM-2 and LGE $>50 \%$, and 385 (27\%) were WM-1 and LGE $>50 \%$. None of the segments in the last group had a transmural LGE extension of $>75 \%$. On the contrary, WM-3 and -4 were associated with LGE $<50 \%$ in 1896 segments $(15 \%$ of total segments) and with LGE $>50 \%$ in 578 segments (5\% of total segments). The group without CEs had a higher number of CT-F segments at follow-up than the $\mathrm{CE}$ group $(P=0.02)$. There was strong intraobserver consistency (ICC $=0.93,95 \%$ CI $0.91-0.94, P<0.001)$ and interobserver consistency (ICC $=0.92,95 \%$ CI $0.91-0.94$, $P<0.001$ ).

During follow-up (median 2.5 years, IQR 1.1 to 4.7 ), 123 patients $(17 \%)$ had CEs (68 cardiac-related deaths and 55 appropriate ICD shocks).

\section{Predictors of cardiac death}

At univariate analysis, age, LV volumes, LVEF, WMSI, extent of LGE, segments with transmural extent $>50 \%$, and CT-F segments-both when considered as a continuous variable (Table 2) or as dichotomic variables (Table 3) - were associated with CEs. 
Table 1 Clinical characteristics and cardiovascular magnetic resonance (CMR) variables in entire population and in subgroups with and without cardiac events

\begin{tabular}{|c|c|c|c|c|}
\hline & $\begin{array}{l}\text { Entire population } \\
(n=730)\end{array}$ & $\begin{array}{l}\text { Cardiac events } \\
(n=123)\end{array}$ & $\begin{array}{l}\text { No cardiac events } \\
(n=607)\end{array}$ & $P$-value \\
\hline Age (years) & $63 \pm 12$ & $66 \pm 10$ & $63 \pm 12$ & 0.002 \\
\hline Male sex (\%) & 88 & 87 & 88 & 0.349 \\
\hline Family history of CAD (\%) & 48 & 43 & 49 & 0.063 \\
\hline Hypertension (\%) & 58 & 59 & 58 & 0.151 \\
\hline Diabetes (\%) & 27 & 28 & 27 & 0.878 \\
\hline Hypercholesterolemia (\%) & 59 & 60 & 59 & 0.974 \\
\hline No. of stenosed vessels & $1.8 \pm 0.9$ & \pm 0.9 & $1.8 \pm 0.9$ & 0.09 \\
\hline Beta-blocker (\%) & 79 & 87 & 77 & 0.06 \\
\hline Diuretics (\%) & 46 & 69 & 40 & $<0.001$ \\
\hline ACE-I/ARB (\%) & 80 & 84 & 79 & 0.357 \\
\hline ANTI ALD (\%) & 30 & 46 & 26 & $<0.001$ \\
\hline$>1 \mathrm{Ml}(\%)$ & 16 & 25 & 14 & 0.005 \\
\hline ICD (\%) & 24 & 54 & 18 & $<0.001$ \\
\hline \multicolumn{5}{|l|}{ CMR data } \\
\hline LVEDV $\left(\mathrm{ml} / \mathrm{m}^{2}\right)$ & $111 \pm 41$ & $136 \pm 46$ & $106 \pm 38$ & $<0.001$ \\
\hline $\operatorname{LVESV}\left(\mathrm{ml} / \mathrm{m}^{2}\right)$ & $71 \pm 42$ & $99 \pm 43$ & $65 \pm 39$ & $<0.001$ \\
\hline LVEF (\%) & $41 \pm 16$ & $30 \pm 10$ & $43 \pm 16$ & $<0.001$ \\
\hline LV mass index & $77 \pm 22$ & $82 \pm 24$ & $76 \pm 21$ & 0.02 \\
\hline LVEF $<30 \%(\%)$ & 29 & 55 & 24 & $<0.001$ \\
\hline Dilated LVEDVi & 39 & 67 & 34 & 0.076 \\
\hline WMSI & $1.7 \pm 0.5$ & $2.0 \pm 0.5$ & $1.7 \pm 0.5$ & $<0.001$ \\
\hline WMSI > $1.7(\%)$ & 52 & 48 & 62 & $<0.001$ \\
\hline LGE extent (\% of LV mass) & $14 \pm 8$ & $16 \pm 8$ & $14 \pm 8$ & 0.003 \\
\hline LGE extent $>$ median & 50 & 62 & 47 & 0.003 \\
\hline Segments with LGE & $6.0 \pm 3.2$ & $6.7 \pm 3.4$ & $5.9 \pm 3.2$ & 0.02 \\
\hline LGE transmural extent 1-50\% (no. of segments) & $2.2 \pm 2.5$ & $2.1 \pm 2.4$ & $2.2 \pm 2.5$ & 0.61 \\
\hline LGE transmural extent $51-100 \%$ (no. of segments) & $3.8 \pm 2.9$ & $4.6 \pm 3.1$ & $3.7 \pm 2.8$ & 0.002 \\
\hline CT-F tissue (no. of segments) & $1.9 \pm 2$ & $1.5 \pm 1.7$ & $2.0 \pm 2.0$ & 0.02 \\
\hline
\end{tabular}

ACE-I, angiotensin converting enzyme inhibitor; aldosterone antagonists; ARB, angiotensin receptor blocker; CAD, coronary artery disease; CMR, cardiac magnetic resonance; CT-F, contractile fibrotic; ICD, implantable cardioverter defibrillator; LGE, late gadolinium enhancement; LV, left ventricular; LVEDV, left ventricular end-diastolic volume; LVEDVi, left ventricular end-diastolic volume indexed; LVEF, left ventricular ejection fraction; LVESV, left ventricular end-systolic volume; MI, myocardial infarction; WMSI, wall motion score index

CT-F segments (chi-square 28.1) had an incremental predictive value $(P<0.001)$ compared to segments with transmural extent $>50 \%$ (chi-square 7.7 ). Also, at multivariate analysis, selected identical parameters-both when considered as continuous (Table 2) or dichotomic (Table 3) variables-were independent predictors of CEs. Particularly, age, LVEF, WMSI, and dilated LVEDV indexed were independent predictors of worse outcome, whereas the presence of CT-F myocardium was the only predictor of better outcome (Additional file 1: Tables S1-S3).

On survival analysis (Fig. 2A), patients with CT-F tissue $(\mathrm{n}=535,75 \mathrm{CEs})$ had better prognosis (HR for $\mathrm{CEs}=0.48,95 \% \mathrm{CI} 0.33-0.68, P<0.001)$ than those without (195 patients, $48 \mathrm{CEs}$ ).
Patients with the presence of CT-F myocardium had a higher cumulative survival at 2,5 , and 7 years than those without (Table 4).

The estimated Kaplan-Meier survival curves in highrisk patients (age $>65$ years, WMSI $>1.7$, and $\mathrm{LVEF}<30 \%$ [Fig. 2B] or LVEDV indexed $>112 \mathrm{ml} / \mathrm{m}^{2}$ [Fig. 2C]) showed that the presence of CT-F tissue is associated with a better prognosis than seen in those without CT-F.

A six-unit risk score was computed based on the results of the stepwise Cox regression analyses. Specifically, three points were assigned to a WMSI $>1.7$, one point to age $>65$ years, one point to either an LVEF $<30 \%$ or a dilated LVEDV $\left(>112 \mathrm{ml} / \mathrm{m}^{2}\right)$, and one point to the absence of CT-F. Subjects were then 
Table 2 Hazard ratios of continuous variables for cardiac events (cardiac death and appropriate ICD shocks) at univariate and multivariate analysis in patients with previous myocardial infarction

\begin{tabular}{llll}
\hline Variable & Univariate & Multivariate model 1 (EF) & Multivariate model 2 (EDV) \\
\hline Age (years) & $1.028(1.011-1.045)^{\mathrm{a}}$ & $1.032(1.015-1.050)^{\mathrm{b}}$ & $1.028(1.011-1.046)^{\mathrm{a}}$ \\
$>1$ AMI & $1.560(0.991-2.456)$ & & \\
MI to CMR interval & $1.007(0.970-1.045)$ & & \\
Post-CMR coronary revascularization & $1.225(0.846-1.773)$ & $1.007(1.003-1.011)^{\mathrm{a}}$ & $0.962(0.946-0.978)^{\mathrm{b}}$ \\
LVEDV ml/m $^{2}$ & $1.009(1.005-1.012)^{\mathrm{b}}$ & & \\
LVESV ml/m $^{2}$ & $1.009(1.006-1.012)^{\mathrm{b}}$ & & \\
LVEF & $0.954(0.941-0.967)^{\mathrm{b}}$ & & \\
LV Mass index & $0.114(0.998-1.016)$ & & \\
WMSI & $2.801(1.949-4.024)^{\mathrm{b}}$ & & \\
No. segments with LGE & $1.029(0.977-1.084)$ & & $0.516(0.357-0.747)^{\mathrm{b}}$ \\
No. segments with LGE 1-50\% & $0.934(0.867-1.045)$ & & \\
No. segments with LGE $>50 \%$ & $1.087(1.025-1.153)^{\mathrm{a}}$ & & \\
LGE extent (\% of LV mass) & $1.025(1.005-1.045)^{\mathrm{c}}$ & & \\
CT-F & $0.858(0.772-0.955)^{\mathrm{a}}$ & & \\
\hline
\end{tabular}

Data presented as hazard ratio $(95 \% \mathrm{Cl})$

a $<0.01$

b $<0.001$

c $<0.05$

Table 3 Hazard ratios of dichotomic variables for cardiac events (cardiac death and appropriate ICD shocks) at univariate and multivariate analysis in patients with previous myocardial infarction

\begin{tabular}{llcc}
\hline Dichotomic variable & Univariate & $\begin{array}{l}\text { Multivariate model 1 } \\
\text { (LVEF <30\%) }\end{array}$ & $\begin{array}{c}\text { Multivariate } \\
\text { model 2 (LVEDV } \\
\text { dilated) }\end{array}$ \\
\hline Age $>65$ years & & $1.649(1.149-2.367)^{\mathrm{a}}$ & $1.664(1.161-2.386)^{\mathrm{a}}$ \\
LVEF $<30 \%$ (cut-off for severe) & $1.65(1.157-2.372)^{\mathrm{a}}$ & $1.956(1.338-2.860)^{\mathrm{a}}$ & $2.047(1.351-3.103)^{\mathrm{a}}$ \\
Dilated LVEDV $\left.>112 \mathrm{ml} / \mathrm{m}^{2}\right)$ & $2.682(1.878-3.829)^{\mathrm{a}}$ & & $2.066(1.289-3.310)^{\mathrm{a}}$ \\
WMSI $>1.7$ (median) & $2.796(1.916-4.080)^{\mathrm{b}}$ & $2.235(1.413-3.534)^{\mathrm{a}}$ & \\
LGE $>13 \%$ (median) & $3.210(2.094-4.919)^{\mathrm{b}}$ & & $0.516(0.359-0.742)^{\mathrm{b}}$ \\
Segments with $>50 \%$ LGE $(\geq 3$ segments) & $1.611(1.120-2.319)^{\mathrm{c}}$ & & $0.538(0.374-0.774)^{\mathrm{a}}$ \\
Presence of CT-F myocardium & $1.533(1.075-2.185)^{\mathrm{c}}$ & &
\end{tabular}

Data presented as hazard ratio $(95 \% \mathrm{Cl})$

a $<0.01$

b $<0.001$

$c<0.05$

divided into four groups based on the values of their individual risk score, which allowed the identification of subsets of patients at no risk (score 0, blue), low risk (score 1-2, green), moderate risk (score 3-4, orange) and high risk (score 5-6, red) (Fig. 3, Additional file 1: Table S4). Figure 3 shows the Kaplan-Meier curves for the four categories of risk score, calculated with two models, one using LVEF (Fig. 3A) and the other LVEDV (Fig. 3B). As shown in Fig. 3, a significantly better event-free survival was observed in score 0 than in the other scores; similarly, a worse prognosis was observed in patients with a higher score in respect to those having a lower score (score 5-6 vs score 3-4; score 3-4 vs score 1-2).

\section{Discussion}

The main finding of our study is that the presence of partially or fully preserved contractility in partially scarred myocardium in patients with previous MI is 


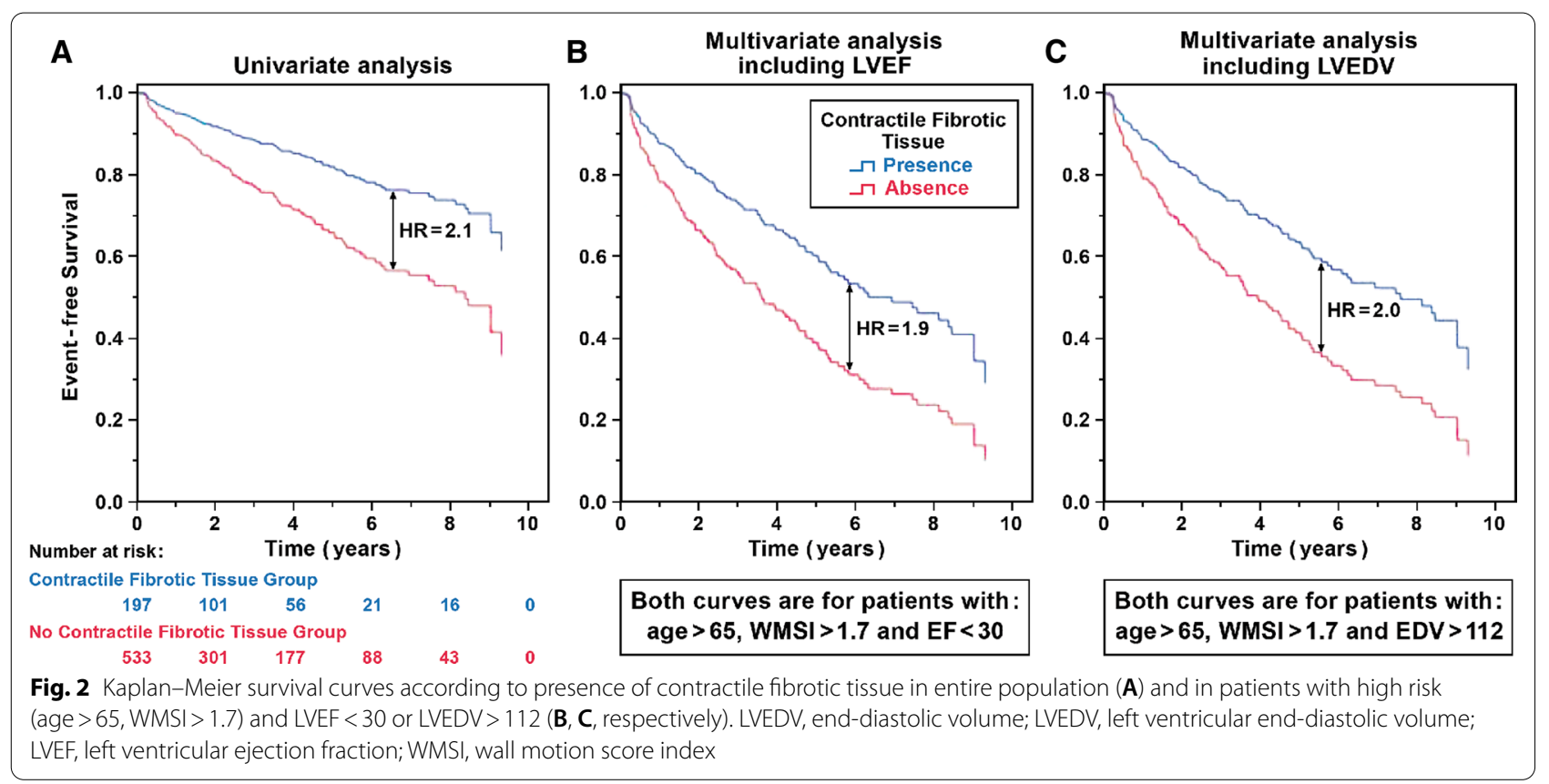

Table 4 Survival at 2 years, 5 years, and 8 years according to CT-F

\begin{tabular}{llll}
\hline $\begin{array}{l}\text { Follow-up } \\
\text { (years) }\end{array}$ & $\begin{array}{l}\text { Lack of CT-F } \\
\text { myocardium (\%) }\end{array}$ & $\begin{array}{l}\text { Presence of CT-F } \\
\text { myocardium (\%) }\end{array}$ & Overall (\%) \\
\hline 2 & 82.5 & 92.0 & 89.5 \\
5 & 65.9 & 82.0 & 77.7 \\
7 & 58.9 & 74.4 & 70.2 \\
\hline
\end{tabular}

CT-F, contractile fibrotic

independently related to better outcome, with fewer CEs at follow-up. Also, in patients at high risk of cardiac death, including those $>65$ years, high WMSI, and severe LV dysfunction ( $\mathrm{LVEF}<30 \%$ ) or increased LVEDV, it has a positive prognostic value. The strength of this study is that we used comprehensive CMR to assess both WM and myocardial fibrosis, reflecting two faces of the same medal, i.e., myocardial viability. WM, as a viability marker, is used by echocardiography and CMR both at rest and after functional or pharmacological stress tests, whereas fibrosis, reflecting the amount of irreversibly damaged myocardium, is typically shown by LGE CMR [5]. The various approaches to predicting functional recovery have differing predictive value, with markers of contractility having the highest positive predictive value for prediction of functional recovery $[15$, 16]. To appreciate the degree of scar transmurality, we used LGE CMR. In the landmark paper by Kim et al., $78 \%$ of dysfunctional segments with no scar at LGE improved contractility after revascularization compared with $55 \%$ of those with $<50 \%$ transmural scar extent and only $2 \%$ of the segments with $>75 \%$ transmural scar extent [10]. Therefore, a cut-off of $50 \%$ of transmural extent of scar tissue is actually one of the CMR criteria of myocardial viability [5, 17-20].

Indeed, using this CMR definition of myocardial viability, Pegg et al. showed that the amount of viable and normal tissue ( $\geq 10$ segments) was predictive of functional improvement after revascularization [20].

More recently, Kwon et al. used this parameter to define jeopardized myocardial segments, concluding that these segments were predictive of mortality when revascularization was not performed [19]. In contrast to the above-mentioned studies, in which the CMR criterion of myocardial segmental viability was based on the presence of $<50 \%$ transmural extent of fibrosis only, we added the evaluation of resting contractility in the same segment. However, because we assessed the patients in the chronic phase of post-MI, no predictions can be made with regard to contractility status immediately after acute MI. Thus, dysfunctional segments in the acute phase may be able to recover substantially after effective revascularization and/or medical therapy.

Prediction of functional recovery in the presence or absence of necrosis was not the goal of this study. In fact, the true novelty of our study was the assessment of the prognostic role of the combined presence of fibrosis and contractility in the same segment.

This is important because segments with non-transmural extent of fibrosis, according to the CMR criteria, are not a homogenous group since functional recovery 

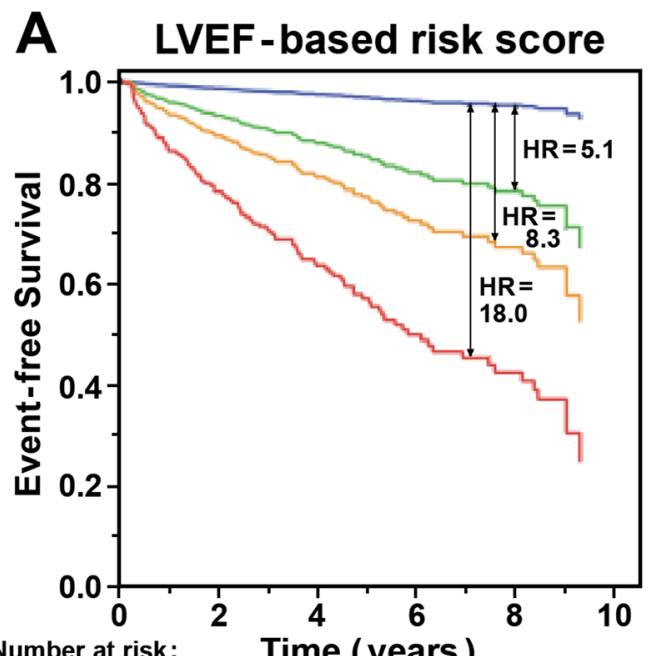

\begin{tabular}{cccccc} 
Number at risk: & \multicolumn{5}{c}{ Time (years ) } \\
$\begin{array}{c}\text { Group 0 } \\
138\end{array}$ & 78 & 47 & 25 & 13 & 0 \\
Group 1-2 & & & & & \\
204 & 111 & 61 & 24 & 14 & 0 \\
Group 3-4 & & & & & \\
227 & 124 & 78 & 38 & 18 & 0 \\
Group 5-6 & & & & & \\
162 & 89 & 47 & 22 & 14 & 1
\end{tabular}

B LVEDV-based risk score

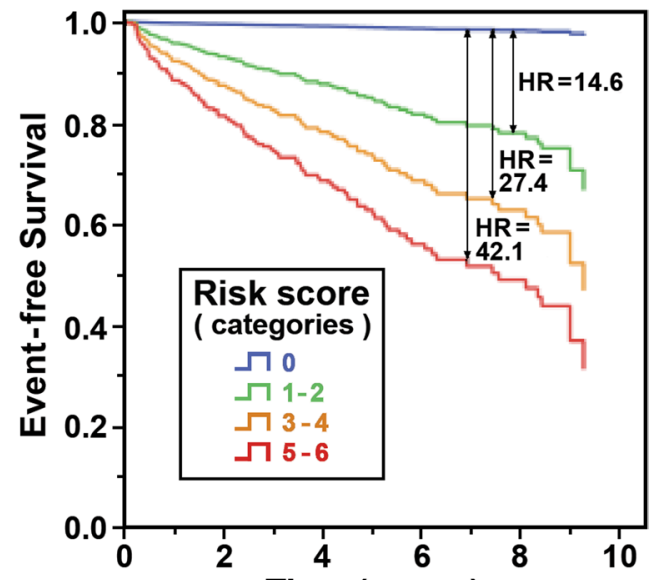

Number at risk:

$\begin{array}{ll}\text { Group }{ }^{0} & \\ & \end{array}$

Group 1-2

Group 3-4

Group 5-6

Time (years)

$\begin{array}{lllll}71 & 45 & 25 & 13 & 0 \\ 114 & 62 & 24 & 14 & 0 \\ 116 & 67 & 34 & 15 & 0 \\ 101 & 59 & 28 & 17 & 1\end{array}$

Fig. 3 Kaplan-Meier survival curves according to six-unit risk score computed on stepwise Cox regression analyses generating groups. The four groups based on risk score — no risk (score 0, blue), low risk (score 1-2, green), moderate risk (score 3-4, orange), and high risk (score 5-6, red) — have considered left ventricular ejection fraction (A, LVEF) and end-diastolic volume (B, LVEDV), respectively

occurs in a portion of them. Indeed, in the study of Pegg et al., about $63 \%$ of segments with $<50 \%$ transmural extent of necrosis had recovered function [20].

In regard to the results of our study, the presence of contractility in the partially scarred segments highlights the importance of segmental function in the prognostic stratification of patients with previous MI. This result confirms previous findings showing the independent prognostic role of WMSI in the prediction of CEs in patients with previous MI [14, 21-24].

In our study, the weight of segmental function/fibrosis was confirmed when CT-F tissue was included in the risk score together with age and other stronger prognostic CMR data, such as severe LV dysfunction and dilated LV.

The improvement of LV regional systolic function is a complex and dynamic phenomenon observed not only after coronary revascularization but also due to many medications [25-29]. Losartan and captopril (echocardiographic sub-study of OPTIMAAL trial) have been shown to be related to an improvement of segmental systolic function detected by a reduced WMSI [25]. As documented in the ANZ carvedilol trial, carvedilol therapy for one year was associated with reduced LV volumes, increased LVEF, and prevented progressive LV dilation in patients with heart failure due to ischemic heart disease [26]. The positive prognostic weight may be linked to several factors including improvement in global LV function [25], favoring reverse remodeling [30] and reducing the risk of arrhythmias [31]. All of these potential mechanisms may be responsible for the positive prognostic role of residual systolic function in post-MI patients. Further, it is intriguing that the positive prognostic weight of contractility (WM-1, -2) counteracts the negative one of myocardial fibrosis, i.e., function is a stronger prognostic variable than fibrosis when they are together in the same myocardial segments. This may account for the evidence that global extent of scar tissue loses its power to predict CEs when added to dilated LVEDV, severely depressed LVEF, and severe impairment of WMSI.

\section{Limitations}

A major limitation of our study is that we do not have data about WM and LGE during the acute phase of MI, and thus we did not assess the possible contractile recovery of the partially fibrotic segments. However, based on previous studies, we supposed that CT-F tissue had a transient and reversible dysfunction due to necrosis and a successive improvement in WM due to acute or chronic revascularization and/or pharmacological data $[10,15$, $16,18,25-29]$. 
Neither the number of CT-F segments per patient nor the different CT-F patterns per each patient were assessed in terms of prognostic weight. Further studies are needed to show the potential prognostic impact of both the number of CT-F segments and the different CT-F patterns.

We did not measure systolic wall thickening. However, we used wall motion analysis, which is a welldefined and accepted semiquantitative method to assess regional systolic function in daily clinical practice, with the aim to assess the prognostic impact of variables used in this context. Also, we did not use a T1 mapping sequence because several examinations were dated and, thus, this sequence was not available. Further, this was a multicenter study using CMR units from different vendors, which would make comparison of T1 mapping data difficult. Moreover, we used semiquantitative methods to assess both WM and transmural extent of LGE. However, one of the objects of this study was to assess the current impact of CMR in daily clinical practice, where LGE technique and WM analysis are largely applied in the context of diagnostic and therapeutic decision-making; in opposition, T1 mapping and quantitative methods of LGE extent and contractility are actually outside the clinical scenario.

\section{Conclusions}

In patients with previous MI, cardiac death is the result of multifactorial elements including clinical, bio-humoral, electrocardiographic, and several imaging variables. In the context of imaging parameters, in addition to adverse prognostic factors (severe LV dysfunction, high WM abnormalities, and dilated LVEDV), the presence of fibrotic segments having contractile activity had an independent protective effect on survival of patients with previous MI. Larger studies are needed to allow generation of a multiparametric score for risk stratification of these patients.

\footnotetext{
Abbreviations

CE: Cardiac event; CMR: Cardiovascular magnetic resonance; CT-F: Contractile fibrotic; ECG: Electrocardiogram; EDV: End-diastolic volume; EF: Ejection fraction; ESV: End-systolic volume; ICC: Intraclass correlation coefficient; ICD: Implantable cardioverter-defibrillator; LGE: Late gadolinium enhancement; LV: Left ventricle/left ventricular; LVEDV: Left ventricular end-diastolic volume; LVEF: Left ventricular ejection fraction; LVESV: Left ventricular end-systolic volume; MI: Myocardial infarction; SD: Standard deviation; WM: Wall motion; WMSI: Wall motion score index.
}

\section{Supplementary Information}

The online version contains supplementary material available at https://doi. org/10.1186/s12968-021-00818-0.
Additional file 1: Table S1. Stepwise analyses, including the C-statistics at each step. Table S2. Hazard ratios of continuous variables for cardiac events (cardiac death and appropriate ICD shocks) within 5 years of follow-up at multivariate analysis in patients with previous MI. Table S3. Hazard ratios of dichotomic variables for cardiac events (cardiac death and appropriate ICD shocks) within 5 years of follow-up at multivariate analysis in patients with previous MI. Table S4. Risk score data distribution (range: $0-6)$ by 4 steps to risk score category with adequate sample size.

\section{Acknowledgements}

From Aurora Cardiovascular and Thoracic Services, Advocate Aurora Health: Jennifer Pfaff and Susan Nord for editorial preparation of the manuscript and Brian Miller and Brian Schurrer for assistance with the figures.

\section{Authors' contributions}

Each author has made substantial contributions to the manuscript. GDB, GDA, $A P, J B, S C$ : conception and design of the work; JB, FP, PP, AB, GC: acquisition and data analysis; AM, GDB, GDA, AP, ME, BK, AB, JB, SC: interpretation of data; GDB, AP: drafted the work or substantively revised it. GDB was a major contributor in writing the manuscript. All authors read and approved the final manuscript.

\section{Funding}

There is no funding to report for this study.

\section{Availability of data and materials}

The datasets used and/or analyzed during the current study are available from the corresponding author upon reasonable request.

\section{Declarations}

Ethics approval and consent to participate

Institutional internal review boards (Comitato Etico Locale dell'Azienda USL Toscana Nord Ovest $-n^{\circ} 266$ ) of the involved hospitals approved this observational and retrospective study. All patients gave informed consent before being enrolled.

\section{Consent for publication \\ Not applicable.}

\section{Competing interests}

The authors declare that they have no competing interests.

\section{Author details}

${ }^{1}$ Clinical and Experimental Department of Medicine, University of Messina, via Consolare Valeria 1, 98100 Messina, Italy. ${ }^{2}$ Fondazione Toscana G. Monasterio, via Giuseppe Moruzzi 1, 56124 Pisa, Italy. ${ }^{3}$ Department of Radiology, KU Leuven - UZ Leuven, Gasthuisberg Campus. Herestraat 49, 3000 Leuven, Belgium. ${ }^{4}$ Department of Information Engineering, University of Pisa, via G. Caruso 16, 56122 Pisa, Italy. ${ }^{5}$ Department of Cardiology, "Santa Maria Dei Battuti" Hospital, Conegliano - ULSS2 Marca Trevigiana, Via Brigata Bisagno 2, 31015 Conegliano, Treviso, Italy. ${ }^{6}$ Cardiac Department, Vannini Hospital Rome, via Acqua Bullicante 4, 00177 Roma, Italy. ${ }^{7}$ Aurora Cardiovascular and Thoracic Services, Advocate Aurora Health, Aurora Sinai/Aurora St. Luke's Medical Centers, 2801 W. Kinnickinnic River Parkway, Ste. 880, Milwaukee, WI 53215, USA. ${ }^{8}$ C.N.R.

Clinical Physiology Institute, via Giuseppe Moruzzi 1, 56124 Pisa, Italy. ${ }^{9}$ Present Address: Department of Cardiology, ISMETT" Hospital, via Ernesto Tricomi, 5,

90127 Palermo, Province of Palermo, Italy.

Received: 27 April 2021 Accepted: 28 September 2021

Published online: 01 November 2021

\footnotetext{
References

1. Pocock SJ, Ariti CA, McMurray JJ, Maggioni A, Køber L, Squire IB, et al. Predicting survival in heart failure: a risk score based on 39372 patients from 30 studies. Eur Heart J. 2013;34:1404-13.
} 
2. Maggioni AP, Dahlström U, Filippatos G, Chioncel O, Crespo Leiro M, Drozdz J, et al. EURObservational Research Programme: regional differences and 1-year follow-up results of the Heart Failure Pilot Survey (ESC-HF Pilot). Eur J Heart Fail. 2013;15:808-17.

3. Cohn JN, Ferrari R, Sharpe N. Cardiac remodeling-concepts and clinical implications: a consensus paper from an international forum on cardiac remodeling. Behalf of an International Forum on Cardiac Remodeling. J Am Coll Cardiol. 2000;35:569-82.

4. Bonow RO, Maurer G, Lee KL, Holly TA, Binkley PF, Desvigne-Nickens P, et al. Myocardial viability and survival in ischemic left ventricular dysfunction. N Engl J Med. 2011;364:1617-25.

5. Bogaert J, Gheysens O, Dymarkowski S, Goetschalckx K. Comprehensive evaluation of hibernating myocardium: use of noninvasive imaging. J Thorac Imaging. 2014;29:134-46.

6. Cwajg JM, Cwajg E, Nagueh SF, He ZX, Qureshi U, Olmos LI, et al. End-diastolic wall thickness as a predictor of recovery of function in myocardial hibernation: relation to rest-redistribution 11-201 tomography and dobutamine stress echocardiography. J Am Coll Cardiol. 2000;35:1152-61.

7. Romero J, Xue X, GonzalezW, Garcia MJ. CMR imaging assessing viability in patients with chronic ventricular dysfunction due to coronary artery disease: a meta-analysis of prospective trials. JACC Cardiovasc Imaging. 2012;5:494-508

8. Bonow RO, Castelvecchio S, Panza JA, Berman DS, Velazquez EJ, Michler $\mathrm{RE}$, et al. Severity of remodeling, myocardial viability, and survival in ischemic LV dysfunction after surgical revascularization. JACC CardiovasC Imag. 2015;8:1121-9.

9. Kelle S, Roes SD, Klein C, Kokocinski T, de Roos A, Fleck E, et al. Prognostic value of myocardial infarct size and contractile reserve using magnetic resonance imaging. J Am Coll Cardiol. 2009:54:1770-7.

10. Kim RJ, Wu E, Rafael A, Chen EL, Parker MA, Simonetti O, et al. The use of contrast-enhanced magnetic resonance imaging to identify reversible myocardial dysfunction. N Engl J Med. 2000;343:1445-53.

11. Aquaro GD, Camastra G, Monti L, Lombardi M, Pepe A, Castelletti S, et al. Reference values of cardiac volumes, dimensions, and new functional parameters by MR: a multicenter, multivendor study. J Magn Reson Imag. 2017:45:1055-67.

12. Positano V, Pingitore A, Giorgetti A, Favilli B, Santarelli MF, Landini L, et al. A fast and effective method to assess myocardial necrosis by means of contrast magnetic resonance imaging. J Cardiovasc Magn Reson. 2005;7:487-94.

13. Aquaro GD, Positano V, Pingitore A, Strata E, Di Bella G, Formisano F, et al. Quantitative analysis of late gadolinium enhancement in hypertrophic cardiomyopathy. J Cardiovasc Magn Reson. 2010;12:21.

14. Di Bella G, Siciliano V, Aquaro GD, Molinaro S, Lombardi M, Carerj S, et al. Scar extent, left ventricular end-diastolic volume, and wall motion abnormalities identify high-risk patients with previous myocardial infarction: a multiparametric approach for prognostic stratification. Eur Heart J. 2013;34:104-11.

15. Wellnhofer E, Olariu A, Klein C, Gräfe M, Wahl A, Fleck E, et al. Magnetic resonance low-dose dobutamine test is superior to SCAR quantification for the prediction of functional recovery. Circulation. 2004;109:2172-4.

16. Baer FM, Theissen P, Schneider CA, Voth E, Sechtem U, Schicha H, et al. Dobutamine magnetic resonance imaging predicts contractile recovery of chronically dysfunctional myocardium after successful revascularization. J Am Coll Cardiol. 1998;31:1040-8.

17. Gerber BL, Rousseau MF, Ahn SA, le de Waroux PJB, Pouleur AC, Phlips $T$, et al. Prognostic value of myocardial viability by delayed-enhanced magnetic resonance in patients with coronary artery disease and low ejection fraction: impact of revascularization therapy. J Am Coll Cardiol. 2012;59:825-35

18. Mahrholdt H, Wagner A, Parker M, Regenfus M, Fieno DS, Bonow RO, et al. Relationship of contractile function to transmural extent of infarction in patients with chronic coronary artery disease. J Am Coll Cardiol. 2003:42:505-12.

19. Kwon DH, Obuchowski NA, Marwick TH, Menon V, Griffin B, Flamm SD, et al. Jeopardized myocardium defined by late gadolinium enhancement magnetic resonance imaging predicts survival in patients with ischemic cardiomyopathy: impact of revascularization. J Am Heart Assoc. 2018;7:e009394.

20. Pegg TJ, Selvanayagam JB, Jennifer J, Francis JM, Karamitsos TD, Dall'Armellina E, et al. Prediction of global left ventricular functional recovery in patients with heart failure undergoing surgical revascularisation, based on late gadolinium enhancement cardiovascular magnetic resonance. J Cardiovasc Magn Reson. 2010;12:56.

21. Mahenthiran J, Das MK, Bhakta D, Ghumman W, Feigenbaum H, Sawada SG. Prognostic importance of wall motion abnormalities in patients with ischemic cardiomyopathy and an implantable cardioverter-defibrillator. Am J Cardiol. 2006;98:1301-6.

22. Shiina A, Tajik AJ, Smith HC, Lengyel M, Seward JB. Prognostic significance of regional wall motion abnormality in patients with prior myocardial infarction: a prospective correlative study of two-dimensional echocardiography and angiography. Mayo Clin Proc. 1986;61:254-62.

23. Yao SS, Qureshi E, Syed A, Chaudhry FA. Novel stress echocardiographic model incorporating the extent and severity of wall motion abnormality for risk stratification and prognosis. Am J Cardiol. 2004;94:715-9.

24. Picano E, Sicari R, Landi P, Cortigiani L, Bigi R, Coletta C, et al. Prognostic value of myocardial viability in medically treated patients with global left ventricular dysfunction early after an acute uncomplicated myocardial infarction: a dobutamine stress echocardiographic study. Circulation. 1998;98:1078-84

25. Møller JE, Dahlström U, Gøtzsche O, Lahiri A, Skagen K, Andersen GS, et al. Effects of losartan and captopril on left ventricular systolic and diastolic function after acute myocardial infarction: results of the Optimal Trial in Myocardial Infarction with Angiotensin II Antagonist Losartan (OPTIMAAL) echocardiographic substudy. Am Heart J. 2004;147:494-501.

26. Doughty RN, Whalley GA, Gamble G, MacMahon S, Sharpe N. Left ventricular remodeling with carvedilol in patients with congestive heart failure due to ischemic heart disease. Australia-New Zealand Heart Failure Research Collaborative Group. J Am Coll Cardiol. 1997;29:1060-6.

27. Kompa AR, Lu J, Weller TJ, Kelly DJ, Krum H, von Lueder TG, et al. Angiotensin receptor neprilysin inhibition provides superior cardioprotection compared to angiotensin converting enzyme inhibition after experimental myocardial infarction. Int J Cardiol. 2018;258:192-8.

28. Tsutamoto T, Wada A, Maeda K, Mabuchi N, Hayashi M, Tsutsui T, et al. Effect of spironolactone on plasma brain natriuretic peptide and left ventricular remodeling in patients with congestive heart failure. J Am Coll Cardiol. 2001;37:1228-33.

29. Suzuki G, Morita H, Mishima T, Sharov VG, Todor A, Tanhehco EJ, et al. Effects of long-term monotherapy with eplerenone, a novel aldosterone blocker, on progression of left ventricular dysfunction and remodeling in dogs with heart failure. Circulation. 2002;106:2967-72.

30. Morimoto R, Okumura T, Hirashiki A, Ishii H, Ichii T, Aoki S, et al. Myocardial contractile reserve predicts left ventricular reverse remodeling and cardiac events in dilated cardiomyopathy. J Cardiol. 2017;70:303-9.

31. Killu AM, Mazo A, Grupper A, Madhavan M, Webster T, Brooke KL, et al. Super-response to cardiac resynchronization therapy reduces appropriate implantable cardioverter defibrillator therapy. Europace. 2018:20:1303-11.

\section{Publisher's Note}

Springer Nature remains neutral with regard to jurisdictional claims in published maps and institutional affiliations. 